\section{An Incidental Pulmonary Meningioma Revealing an Intracranial Meningioma: Primary or Secondary Lesion?}

Marco Chiarelli, MD, Matilde De Simone, MD, PhD, Martino Gerosa, MD, Angelo Guttadauro, MD, and Ugo Cioffi, MD, PhD

Department of Surgery, Ospedale Alessandro Manzoni, Lecco; Department of Surgery, University of Milan, Milan; and Department of Surgery, University of Milan-Bicocca, Milan, Italy

A 68-year-old man underwent a resection of the right middle lobe for a solitary lesion detected at computed tomography. The histologic result was suggestive for a pulmonary meningioma. Although the result of a preoperative brain computed tomography scan was negative, magnetic resonance imaging showed a skull-base meningioma. On the basis of the absence of symptoms, we decided to observe the intracranial meningioma. At 3 years of follow-up, the patient was free of recurrence and the cerebral lesion was stable. Primary pulmonary meningioma and benign meningioma metastasis share identical microscopic findings, and only a central nervous system radiologic study allows their distinction. The pulmonary lesion in our patient was classified as a meningioma metastasis.

(Ann Thorac Surg 2015;99:e83-4) (C) 2015 by The Society of Thoracic Surgeons

$\mathrm{M}$ eningioma is a central nervous system (CNS) tumor arising from meningothelial cells of the arachnoid layer of the meninges and generally characterized by slow growing and a favorable prognosis. This type of neoplasm represents approximately $20 \%$ of primary intracranial neoplasms with an annual incidence of 6 per 100,000 [1].

A pulmonary lesion with histologic features of meningioma could be a primary or a secondary tumor. Primary pulmonary meningioma (PPM) is a very rare pathologic entity, defined by the typical histopathologic features of meningioma in the absence of CNS lesions [2]. Extracranial meningioma metastases (MMs) occur in $0.1 \%$ of cases, and the lung is most frequent involved site [3].

We describe a patient with a pulmonary mass initially classified as a primary meningioma on a negative preoperative cranial computed tomography (CT) scan, but subsequently reconsidered as a secondary lesion when magnetic resonance imaging (MRI) performed after the operation showed an intracranial meningioma.

A 68-year-old man presented at our institution for a mass located in the right middle lung lobe detected incidentally

Accepted for publication Jan 16, 2015.

Address correspondence to Dr Chiarelli, Dipartimento di Chirurgia, Ospedale A. Manzoni, Via dell'Eremo 911, 23900 Lecco, Italy; e-mail: mee.chiarelli@gmail.com. by a chest roentgenogram. The patient's medical history was unremarkable and he referred no respiratory or neurologic symptoms.

A chest CT showed a 5.5-cm solitary lesion localized in middle lobe adjacent to the parietal pleura. No further pulmonary lesions or mediastinal adenopathy were detected (Fig 1A).

Brain CT, abdominal CT, and technetium 99m-scintigraphy were negative for primary and secondary lesions. In consideration of the peripheral location of the lesion and the absence of distant metastases, a wedge resection of the middle lobe was performed through a musclesparing axillary thoracotomy. Intraoperative frozen sections were compatible with a pleural solitary fibrous tumor.

The surgical specimen showed a $5.5-\mathrm{cm}$ bilobated gray mass without infiltration of surrounding parenchyma or pleura. Microscopically the neoplasm consisted of polygonal and spindle cells organized in whorls and bundles and surrounded by thin layers of fibrous tissue (Fig 2).

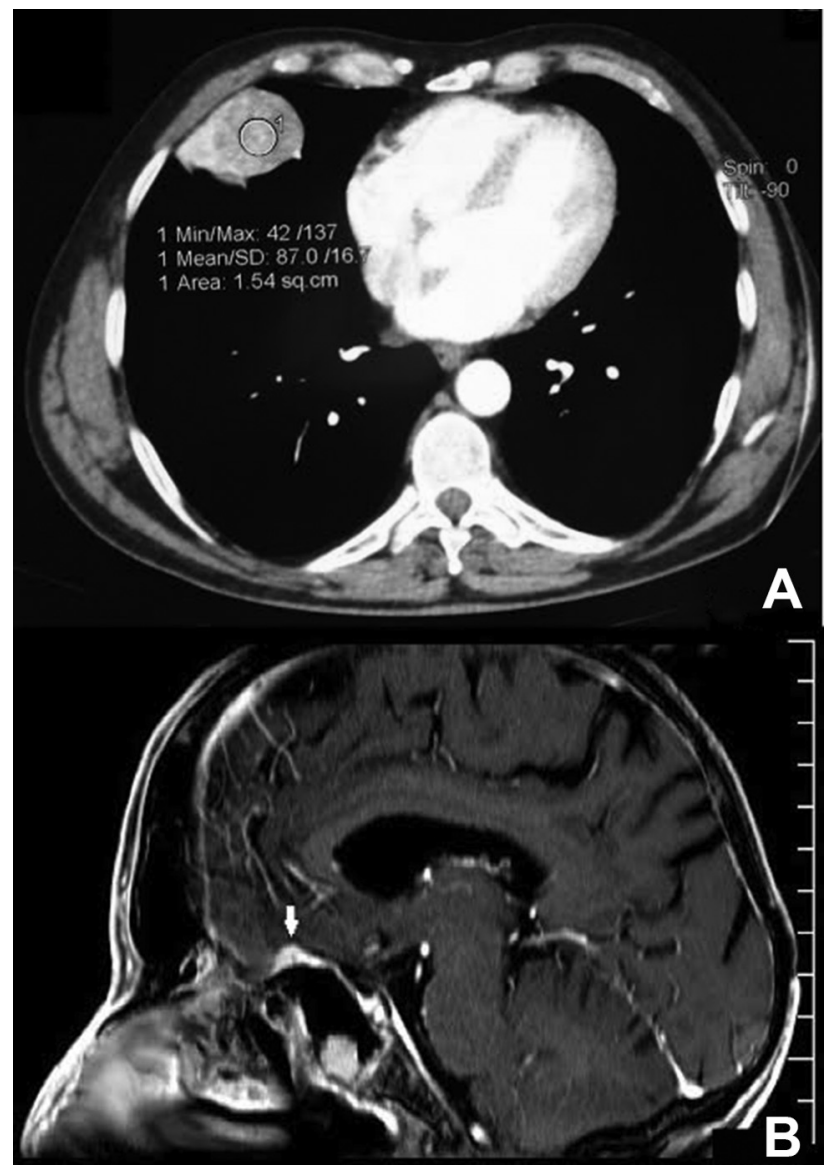

Fig 1. (A) Contrast-enhanced chest computed tomography scan shows a 5.5-cm-diameter subpleural mass located in the middle right lobe. (B) Contrast enhanced T1-weighted brain magnetic resonance imaging shows a en plaque meningioma on planum sphenoidale (arrow), without signs of compression or perilesional edema. 


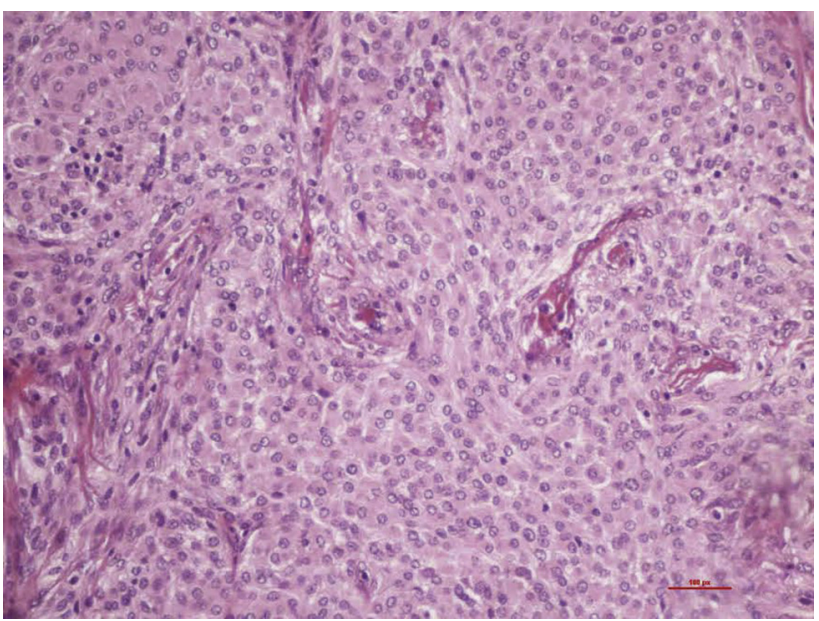

Fig 2. The tumor was characterized microscopically by a proliferation of polygonal and spindle cells organized in bundles and whorls (hematoxylin and eosin staining, $20 \times$ ).

Immunohistochemical staining was positive for vimentin, epithelial membrane antigen, was focally positive for S-100 and negative for cytokeratin AE1/AE3 and CD34. Mitotic count was 1 to 2 mitosis per 10 high-power fields. The MIB-1 labeling index was less than $2 \%$. The morphologic and immunohistochemical features were suggestive for a pulmonary benign meningioma.

To confirm the diagnosis of PPM, a brain and spinal MRI were performed. A previous undetected 1-cm lesion was found in planum sphenoidale. This image was judged compatible with a CNS meningioma (Fig 1B).

On the basis of radiologic findings and the lack of symptoms, we decided to observe the intracranial meningioma. At 3 years of follow-up, the patient was free of local and distant recurrence at total-body $\mathrm{CT}$, and the cerebral lesion was stable on MRI.

\section{Comment}

Ectopic meningiomas are very infrequent and in most cases are localized in the head and neck region. PPM is a rare but well-defined tumor [2, 4]. PPM presents as an asymptomatic solitary lung nodule and generally shows a benign course after surgical excision [2].

This type of neoplasm must meet two diagnostic criteria: the histologic features of a meningioma and the absence of CNS lesion [2]. A radiologic study of the CNS is mandatory to exclude an intracranial or spinal meningioma, and presently, MRI is preferred to CT for its higher sensibility $[1,5]$.

MMs are usually detected after the excision of the CNS lesion and may appear many years after the primary tumor. The occurrence of distant metastasis seems related with malignant histology, venous sinus invasion, and local recurrence [3].

The World Health Organization classification has categorized meningiomas into three grades: grade 1, benign; grade 2, atypical with intermediary features; and grade 3 , anaplastic characterized by malignant histology [1]. Lesions with aggressive features (grade 3 and 2) show a higher metastatic rate, but even histologically benign meningioma (grade 1) can metastasize [5]. Grade 1 MMs present an excellent prognosis, and surgical resection is the treatment of choice [3]. Benign pulmonary MMs can occur in association with primary lung cancer [3]. A complete review by Incarbone and colleagues [6] analyzed all cases of published benign meningioma with a histologic diagnosis, and the coexistence of benign PMM and lung cancer was found in 1 of 25 patients.

PPM and pulmonary benign MM have identical microscopic findings and only a CNS radiologic study allows their distinction.

In our patient the lack of pathologic examination of the intracranial tumor represented a limit, but the pulmonary lesion must be classified as a MM. However the absence of radiologic signs of enlargement at 3 years of follow-up poses the question of whether the two lesions might be synchronous.

Our case also points out the high sensibility of contrast enhancement T1-weighted MRI; indeed, occasionally CT could not detect en plaque skull-base meningiomas [1].

In conclusion, complete surgical resection is basic for diagnosis and allows a definitive treatment in both PPM and pulmonary benign MM $[2,3]$.

We thank Dr Gerardo Cioffi, native speaker, for reviewing the English language.

\section{References}

1. Whittle IR, Smith C, Navoo P, Collie D. Meningiomas. Lancet 2004;363:1535-43.

2. Picquet J, Valo I, Jousset Y, Enon B. Primary pulmonary meningioma first suspected of being a lung metastasis. Ann Thorac Surg 2005;79:1407-9.

3. Cheng YJ, Wu JT, Chen HY, et al. Coexistence of intracranial meningioma, pulmonary meningiomas, and lung cancer. Ann Thorac Surg 2011;91:1283-5.

4. Cesario A, Galetta D, Margaritora S, Granone P. Unsuspected primary pulmonary meningioma. Eur J Cardiothorac Surg 2002;21:553-5.

5. Surov A, Gottschling S, Bolz J, et al. Distant metastases in meningioma: an underestimated problem. J Neurooncol 2013;112:323-7.

6. Incarbone M, Ceresoli GL, Di Tommaso L, et al. Primary pulmonary meningioma: report of a case and review of the literature. Lung Cancer 2008;62:401-7. 